\title{
Noninvasive 3D geometry extraction of a Sea lion foreflipper
}

\author{
Chen Friedman $*$ \\ Bryan W. Joel ${ }^{\dagger}$ \\ Allen R. Schult* \\ Megan C. Leftwich* \\ *Department of Mechanical and Aerospace Engineering, \\ The George Washington University, USA \\ Department of Mechanical and Civil Engineering, \\ California Institute of Technology, USA
}

\begin{abstract}
Underwater propulsion that leaves little traceable wake structure while producing high levels of thrust is a highly desired goal. A potential biological model is the California sea lion, a highly maneuverable aquatic mammal that produces thrust primarily with its foreflippers without a characteristic flapping frequency. In an effort to mimic the sea lion flipper, a flipper from a sea lion carcass has been scanned using several non invasive methods with the goal of manufacturing a robotic flipper with identical geometry. The scanning methods are hereby compared for overall quality, accuracy, and level of detail captured.
\end{abstract}

Index Terms - Sea lion, Swimming, Foreflippers, Structured Light, 3D Modeling.

\section{INTRODUCTION}

California Sea Lions are very agile swimmers and unlike many marine animals they use their foreflippers rather than hind flipper undulation to generate high thrust values. Their foreflippers feature multiple degrees of freedom which allows them to be used for primary thrust production as well as for stability and control during various maneuvers (turning, rolling, spinning, etc.). For thrust production, the flippers are clapped together in a downward motion until they are adducted to the body, a motion that involves more than one rotation axis which, as mentioned above, is different than either lateral tail undulation (fish) or vertical tail flapping (mammals). The clap motion essentially has no dominant frequency as it is accompanied by a prolonged glide, unlike the characteristic flapping frequency that is present in the wake of a constantly undulating flipper $[1,2]$. The glide phase is also characterized by a relatively low drag coefficient $[3,4]$.

A limited amount of qualitative studies show that the flippers are used both for thrust, stability, and control during the motion [5]. Quantitative studies mainly measured cost of transport [6], drag [3], and banked turn performance [7]. The available morphometric data includes weight, overall size (length, girth, surface area, etc.), and volume [3, 4, 8].

Recently the kinematics of a California sea lion flipper during the thrust phase was extracted using video tracking [9].

${ }^{\ddagger}$ Corresponding author: The George Washington University, Science and Engineering Hall, 800 I St NW, Washington, DC 20052, USA, chen.friedman1@gmail.com
However, advanced analysis requires a more detailed geometric description of the flipper, including quantities such as span, aspect ratio, chord and thickness distributions, airfoil shapes along the span, and skin surface patterns. For this purpose, a severed sea lion flipper was scanned using three non invasive methods:

1. High resolution structured light based scanner: both Artec $\mathrm{Eva}^{\mathrm{TM}}$ and Artec Spider ${ }^{\mathrm{TM}}$.

2. Low resolution structured light based scanner, based on Microsoft's Kinect ${ }^{\mathrm{TM}}$ sensor [10].

3. Image based software capable of producing 3D models: 123DCatch by Autodesk ${ }^{\odot}$.

The high resolution structured light approach has the advantage of better accuracy, although at the expense of a considerably higher price and required experience level (see Table 1 for more details). The three approaches are compared with respect to capturing detailed features such as trailing edge shape, skin wrinkles (which may play an role in flow over the flipper) and overall surface resolution. Additionally, airfoil sections are extracted using perpendicular planar intersections in the chordwise direction. Airfoils provide necessary information for flipper performance modeling and simulations as part of future work. The flipper area that was scanned is shown in Figure 1.

Structured light is a form of image processing where several light patterns are projected onto an object while a camera records the image as seen on the projected surfaces. The bent light patterns are analyzed to extract the shape of the object on which the light is projected [11]. These methods gained recent attention as it is not only a non invasive method, but is also considered to be safer as no laser scanners are involved. The cameras used typically record video at 30 frames per second and the data is analyzed with the same frequency.

\section{EXPERIMENTAL SETUP}

\section{A. Sea Lions}

The Smithsonian National Zoological Park (SNZ) is home to three non-research, female California sea lions (Zalophus Californianus). Recently, a fourth individual died unexpectantly, and so her flippers were used for this investiga- 


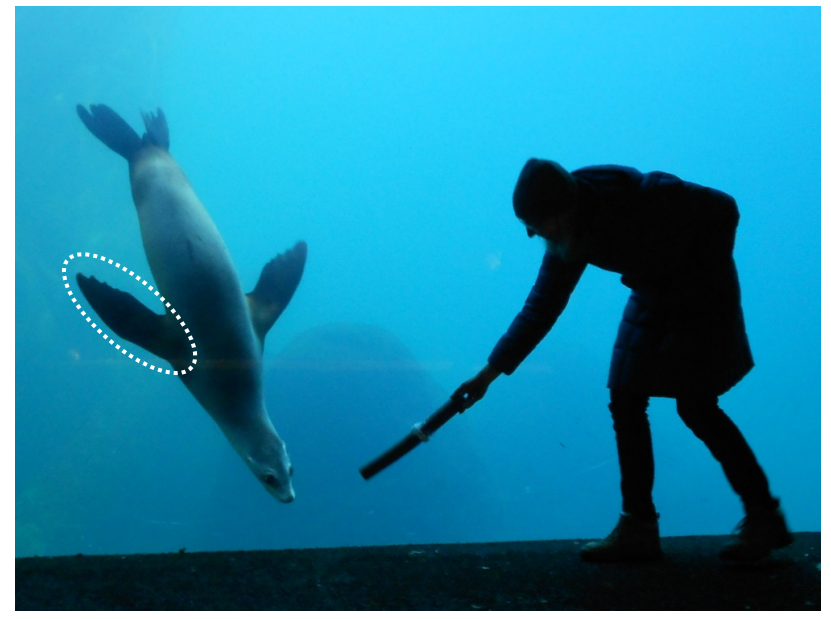

Figure 1: The fore flipper of a California sea lion is highlighted with the white oval. It is approximately $1 / 4$ the body length of the entire animal's extended length.

tion. The flipper was hung at the SNZ necropsy lab and was scanned using three non invasive approaches detailed below.

\section{B. Scanning Approaches}

The three methods used in this work are summarized below:

1. High-resolution Structured Light Scanner: The flipper was first scanned using the Artec Eva ${ }^{\mathrm{TM}}$ (Artec 3D), a portable scanner based on structured light technology. Results from several scans were fused together with additional scans using the Artec SpiderTM which has a higher spacial resolution and produces a highly detailed three dimensional model. The Artec Eva resolution is listed as $0.5 \mathrm{~mm}$ with an accuracy of $0.1 \mathrm{~mm}$, while the Artec Spider is listed with a resolution of $0.1 \mathrm{~mm}$ and accuracy of up to $0.01 \mathrm{~mm}$.

2. Low-resolution Structured Light Scanner: The Kinect $^{\mathrm{TM}}$ sensor from Microsoft is used for image tracking and may also be used to scan objects into 3D models [10]. This sensor also uses structured light technology. The resolution of the sensor is considerably lower than that of the Artec sensors and is expected to yield a relatively less detailed model. The camera has a listed resolution of 1280x960.

3. Image Processing Software: For the image processing approach, we used 123DCatch, a free online application by Autodesk Inc ${ }^{\circledR}$ which receives a set of pictures as an input and processes them into a three dimensional model defined as triangular surface elements. The output consists of a mesh of triangles and includes the color scheme of the object. The application also allows for limited model manipulation and editing. The pictures were taken with a Nikon D3100 camera with an 8MP resolution.

\section{Scaling}

The low resolution structured light and the image based methods produce a model that is not to scale, and therefore require an additional post processing step. An unique object with a well defined length needs to be included in the output of both these methods in order to allow accurate post processing scaling. The flipper itself did not have a well defined accurate points of measurement that allowed accurate scaling (definitions of points along the flipper may be subjective). However, the high-resolution structured light method does produce a scaled 3D model since it uses multiple sources of light during the scanning process with a known distance between them. This advantage is not small as it relieves the output model from the requirement to contain an additional object for scaling.

\section{Background Objects}

All three methods scanned the flipper while it was hung vertically. Objects in the background were also captured in the scan process as the scanners do not distinguish between the target object and its surroundings. These objects need to be removed in post processing. Therefore, background objects need to be within sufficiently large distance so they would be distinguishable during the post processing cleaning process.

\section{E. Flipper Thickness Ratio}

The flipper thickness is an order of magnitude smaller than its chord, which generally creates problems with most shapecapturing methods. The reason is that these methods are based on tracking and matching sequentially captured frames to create the 3D model. Therefore, the transition from one flipper side to the other causes a problem when there's very little data to match frames which capture both flipper sides. The flipper thickness does not provide sufficient features for continuous matching, causing the scanner to loose tracking. This issue posed a challenge for all three methods although it was overcome by the high resolution scanner, as explained below.

\section{F. Methods Comparison}

All three methods produce a point cloud that can be opened and manipulated on current 3D modeling software. Table 1 presents a comparison of the different approaches's advantages and disadvantages with respect to accuracy, resolution, cost, the amount of post processing work required, etc. The authors believe that for the case of the sea lion flipper, with the challenges described above, the high resolution method seems to be favorable despite the significantly higher price. The other methods produced a significantly lower detailed model which is not adequate for the intended research purposes. 
Table 1: Comparison of all three scanning methods.

\begin{tabular}{|c|c|c|c|c|c|c|c|}
\hline Method & Accuracy & $\begin{array}{l}\text { Detail Cap- } \\
\text { tured }\end{array}$ & $\begin{array}{l}\text { Post Pro- } \\
\text { cessing }\end{array}$ & $\begin{array}{l}\text { Training } \\
\text { Required }\end{array}$ & $\begin{array}{l}\text { Equipment } \\
\text { Required }\end{array}$ & Cost & Success \\
\hline $\begin{array}{l}\text { Structured } \\
\text { Light, } \\
\text { High- } \\
\text { resolution }\end{array}$ & $\begin{array}{l}\text { very high } \\
O(0.1) \\
\text { mm }\end{array}$ & $\begin{array}{l}\text { up to fur } \\
\text { hair surface } \\
\text { texture }\end{array}$ & $\begin{array}{l}\text { Significant } \\
\text { compu- } \\
\text { tational } \\
\text { power }\end{array}$ & $\begin{array}{l}\text { highly } \\
\text { trained } \\
\text { user } \\
\text { required }\end{array}$ & $\begin{array}{l}\text { high detail } \\
\text { scanner }\end{array}$ & $\begin{array}{l}\text { expensive } \\
(O(\$ 1000))\end{array}$ & very successful \\
\hline $\begin{array}{l}\text { Structured } \\
\text { Light, Low- } \\
\text { resolution }\end{array}$ & $\begin{array}{l}\text { medium } \\
\text { level } O(1) \\
\mathrm{mm}\end{array}$ & $\begin{array}{l}\text { relatively } \\
\text { low, gen- } \\
\text { eral surface } \\
\text { curvature }\end{array}$ & $\begin{array}{l}\text { Does not } \\
\text { required } \\
\text { signifi- } \\
\text { cant post } \\
\text { processing }\end{array}$ & $\begin{array}{l}\text { some } \\
\text { training } \\
\text { required }\end{array}$ & $\begin{array}{l}\text { Kinect } \\
\text { sensor and } \\
\text { software }\end{array}$ & $\begin{array}{l}\text { cheap } \\
(O(\$ 100))\end{array}$ & $\begin{array}{l}\text { failed to capture } \\
\text { the full flipper. } \\
\text { only one side can } \\
\text { be captured at a } \\
\text { time }\end{array}$ \\
\hline $\begin{array}{l}\text { Image Pro- } \\
\text { cessing }\end{array}$ & $\begin{array}{l}\text { relatively } \\
\text { low } O(1) \\
\text { mm }\end{array}$ & $\begin{array}{l}\text { relatively low, } \\
\text { fine surface } \\
\text { curvatures }\end{array}$ & $\begin{array}{l}\text { Some post } \\
\text { processing } \\
\text { required }\end{array}$ & $\begin{array}{l}\text { minimal } \\
\text { training } \\
\text { required }\end{array}$ & $\begin{array}{l}\text { any } \\
\text { current } \\
\text { camera }\end{array}$ & $\begin{array}{l}\text { cheap } \\
(O(\$ 100))\end{array}$ & $\begin{array}{l}\text { very successful, } \\
\text { required some } \\
\text { manipulation of } \\
\text { flipper root }\end{array}$ \\
\hline
\end{tabular}

\section{RESULTS}

A high resolution picture of the flipper is presented in Figure 2 alongside the corresponding images of all three scanned flipper models. A short description and relative comparison of the results by all three methods is given below.

\section{A. High Resolution Structured Light Scanner}

The image obtained by the high resolution structured light scanner shows the impressive amount of detail captured. The scan captured bone structure, skin wrinkles, vein marks, and over all flipper silhouette with high detail, as compared to the flipper picture. Qualitatively, compared with the results of the other two methods, this method was found to be the most accurate and was later chosen as the preferred model to further analyze the flipper (for extracting section airfoil shapes and creating a 3D physical model for hydrodynamic studies). The accuracy and resolution of both scanners, as well as the professional post processing created a well defined model suitable for follow on studies on the flipper. This was, of course, at the expense of this method having the highest cost of the three.

The high resolution scanner was able to overcome the flipper thickness ratio problem explained above due to the higher amount of details captured during the scanning process. This method is the only one that could capture both sides of the flipper continuously (without modifying the flipper). This is a major advantage as two separate scans of both sides of the flipper may not have enough overlap to allow stitching them together in post processing (in general, stitching is considered as additional post processing work and may introduce more errors into the finalized model).

\section{B. Flipper Skin Movement Due TO Gravity}

As mentioned above, the high resolution scanner data is obtained using two scanners which provide different degree of detail. Several scans are obtained with each scanner and those are later merged together with an optimization tech- nique during post processing. This step revealed some minuscule geometry changes in certain areas due to gravity pulling down on the flipper's skin folds. This issue was especially pronounced in the root area where the skin was relatively loose despite efforts to keep it connected and immobile. The overall scan time was approximately 30 minutes, for all the scans obtained with both scanners. Note that the previously frozen flipper thaws during the scanning process, which may also contribute to changes on the flipper's surfaces. Both these issues only affected the high resolution scanning method as the other two methods did not have a high enough resolution to discern the small geometry changes.

\section{Low Resolution Structured Light Scanner}

The low resolution Kinect based scanner produced relatively lower quality results without the ability to continuously scan the flipper on both sides. Since the scanner relies on continuous tracking, and its software does not have the capability to fuse several scans together into a single model, this method was limited to scanning one side of the flipper. The low resolution structured light method introduced a considerable amount of noise into the scan, both on the model and around it, as evident by the multiple (although relatively small) artifacts surrounding the flipper edges.

Nonetheless, some of the flippers' fine features were captured by this method, as seen in Figure 2 (c). Relatively large features such as the long sea lion fingers which cause surface ridges and the curviness of the trailing edge were relatively well captured. However, the captured trailing edge seems to be much smoother as compared to the relatively sharper spikes that the original flipper trailing edge exhibits. The surface details also seem to be much better captured by the high resolution structured light scanner (as expected). Quantitatively, this method did not even capture the thickness of the largest finger bones embedded in the flipper, which are approximately $0.5 \mathrm{~cm}$ in size. 

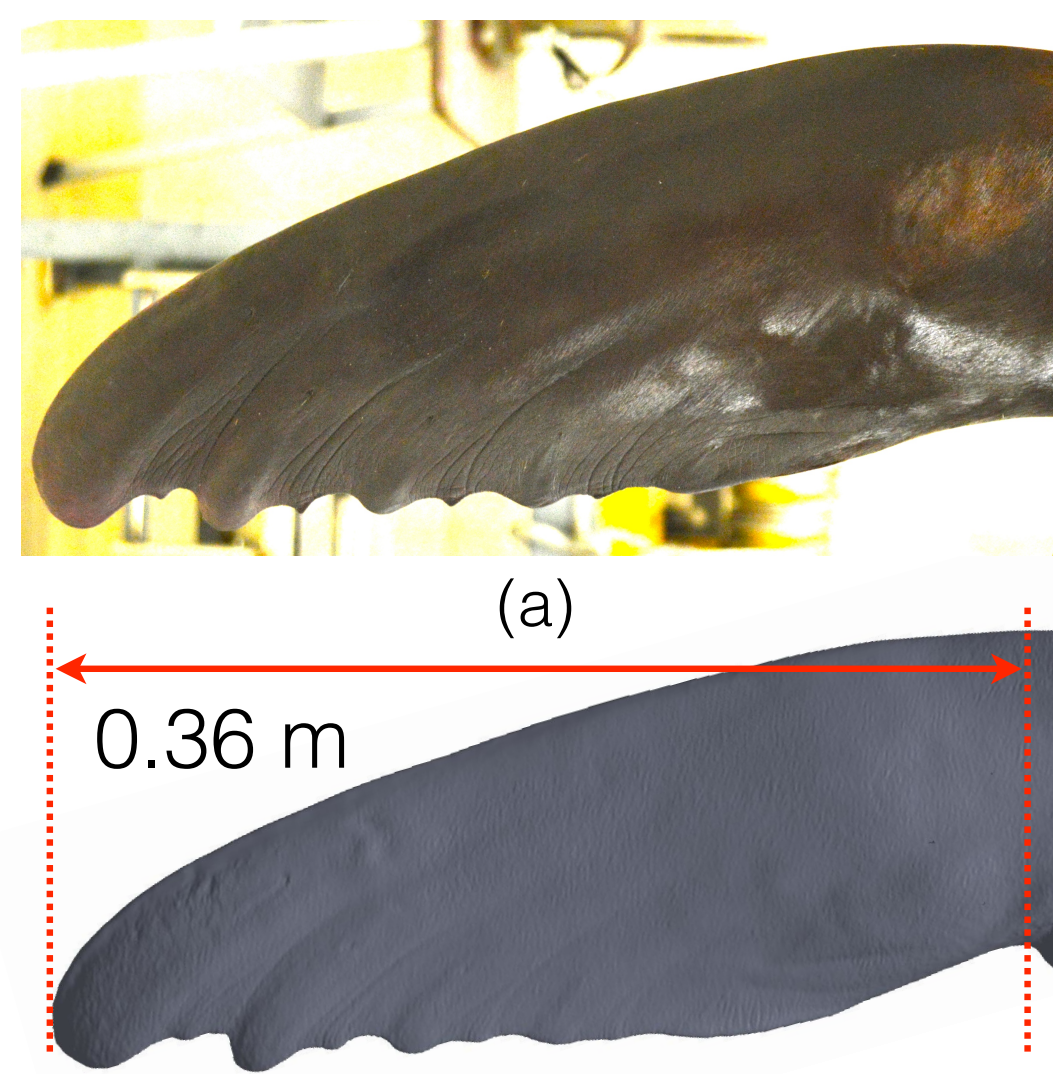

(b)

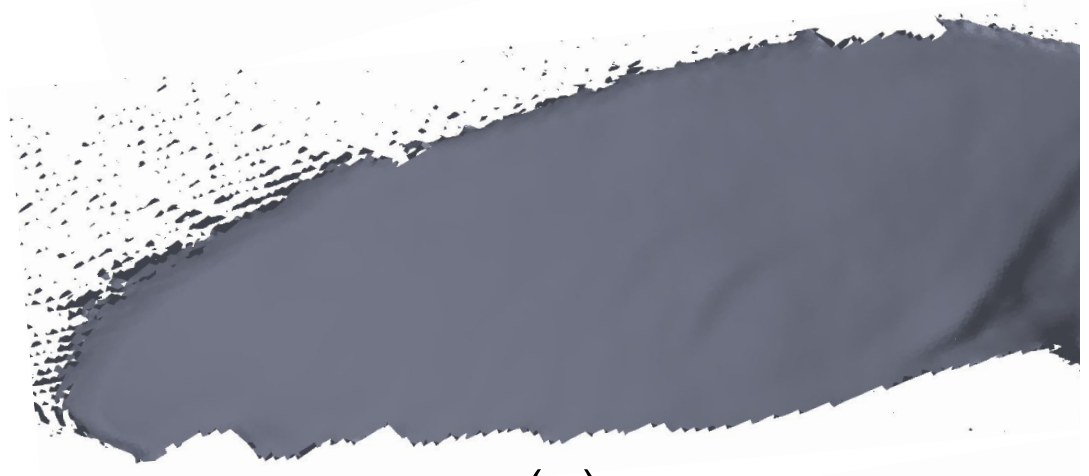

(c)

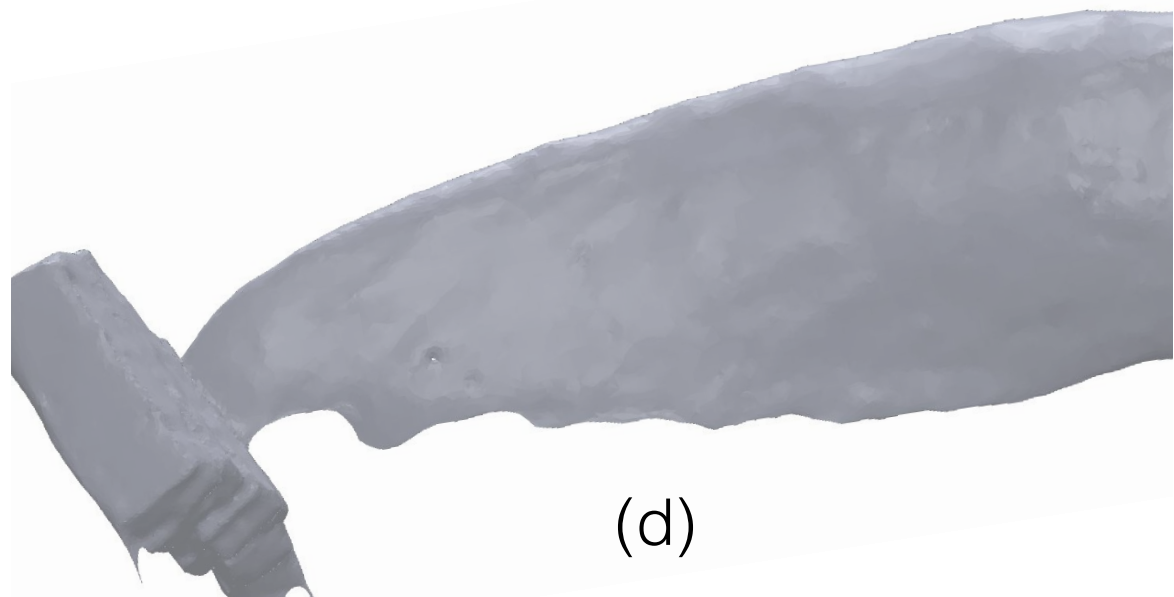

Figure 2: Flipper geometry extraction. (a) Sea lion flipper picture; (b) High resolution structured light scanner; (c) Low resolution structured light scanner; (d) Picture based modeling software. 


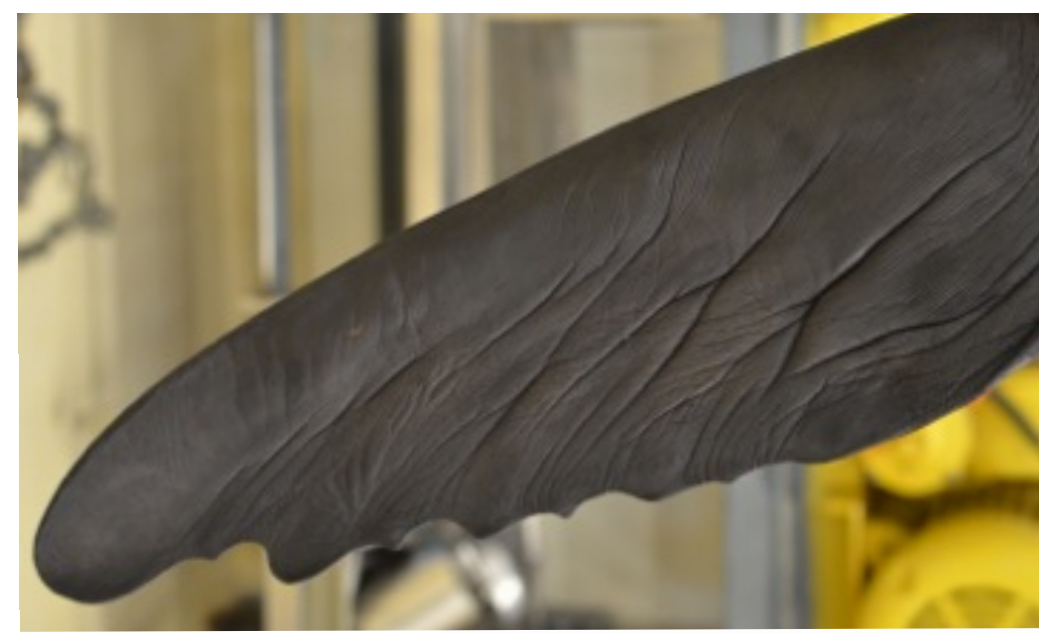

(a)

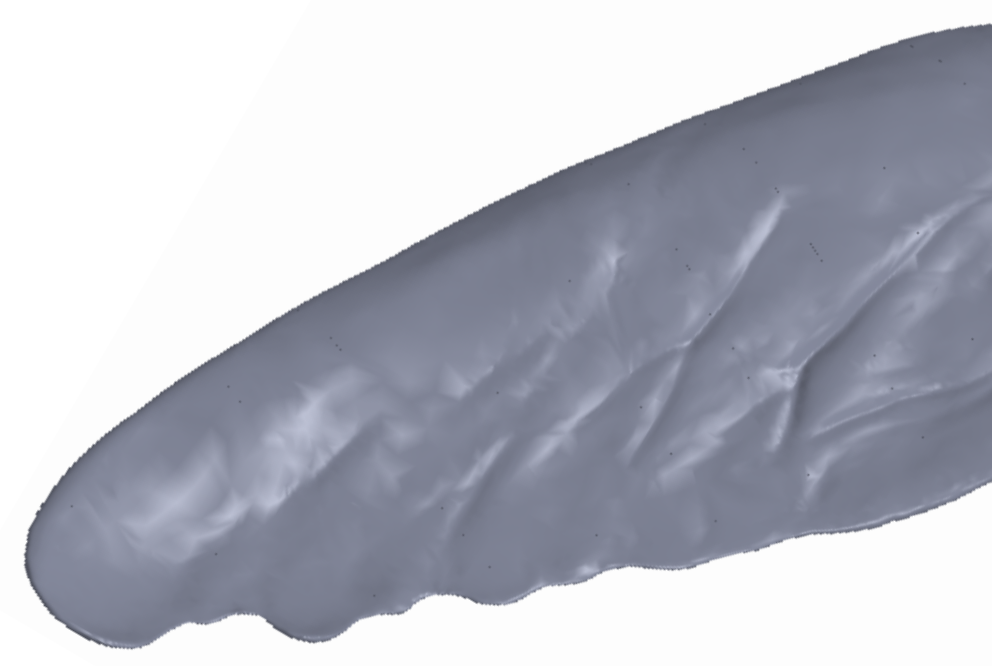

(b)

Figure 3: The inner surface of the sea lion foreflipper shown (a) in a photograph and (b) with data extracted by the high resolution structured light scanner. It includes both large-scale features easely captured by the scanner and finer structures that are sometimes below the scanners resolution.

\section{Image Based Software}

As with the case of the low resolution scanner, the image based software was not able to produce a complete model of the flipper with both sides. However, by adding a thick object at the root and thus increasing the overall effective thickness ratio, the method was capable of producing a full model of both flipper sides. Some post processing work had to be carried out as the flipper surfaces were wrongfully correlated (and therefore connected) to some of the objects in the background of the flipper. Post processing included refining the baseline grid that was produced by the 123DCatch software, and eliminating grid cells that included background objects, keeping only grid cells that portrayed flipper surfaces. It is important to note that some cells contained mixed representations of both background objects as well as flipper surfaces.
The removal of such cells caused much of the rough surfaces that can be seen in Figure 2, in particular the detailed trailing edge silhouette is not well captured using this scanning method (no spikes are visible).

For this method, the flipper was fitted with a foam blocks at tip, which effectively increased the thickness ratio (to approximately 0.5 ) and allowed for a continuous set of pictures on both flipper sides to be merged into a single model. Although the foam blocks at the root do not cover any area of interest, the ones at the tip covered approximately an area of $5 \mathrm{~cm}^{2}$. The area is relatively flat and therefore can be easily removed in post processing [12]. Quantitatively, this method was able to capture only flipper surface details that were larger than approximately $1 \mathrm{~cm}$. 


\section{E. Additional Comparison - High Resolution Scanner}

The scan obtained using the high resolution scanner for the inner surface of the flipper is also compared to the flipper picture, presented in Figure 3. The inner surface is being adducted into the sea lion's body during the glide phase of the swimming cycle, and therefore has more skin wrinkles and surface features relative to the smoother outer surface. Those features are clearly seen in the scanned image with all but the minor ones successfully captured by the high resolution scanner.

\section{F. Airfoil Shapes}

Figure 4 presents an isometric view of the flipper with nine airfoil cross sections that have been extracted from the high resolution scan. The airfoil shapes closely resemble those of typical wings. This supports the claim that the sea lion flipper produces thrust using a lift based mechanism and not a paddling mechanism (unlike seals and walruses despite also being pinnepeds). The extracted airfoils provide the necessary details for flipper modeling and simulations as part of future work. Table 2 provides the local geometrical information for the extracted airfoil cross sections.

Table 2: local airfoil properties.

\begin{tabular}{|c|c|c|}
\hline Cross section \# & Chord $\mathrm{m}$ & Thickness ratio \\
\hline 1 (root) & 0.140 & 0.302 \\
\hline 2 & 0.139 & 0.278 \\
\hline 3 & 0.135 & 0.236 \\
\hline 4 & 0.128 & 0.216 \\
\hline 5 & 0.113 & 0.193 \\
\hline 6 & 0.110 & 0.153 \\
\hline 7 & 0.091 & 0.168 \\
\hline 8 & 0.084 & 0.123 \\
\hline 9 (tip) & 0.056 & 0.113 \\
\hline
\end{tabular}

\section{G. Global Flipper Properties}

Overall flipper properties were extracted from the high detail structured light model, and are given in Table 3. The calculated aspect ratio of 3.34 is similar to values for typical bird wings as expected from the sea lion that generates all its thrust using its pectoral flippers. Note that this value does not include the span of the sea lions body and the other flipper. The flipper root location was determined to be the narrowest flipper chord location, just before the tip of the shoulder muscles.

Table 3: Flipper extracted properties.

\begin{tabular}{l|l} 
Property & measurement \\
\hline \hline Mean Chord & $0.11 \mathrm{~m}$ \\
Semi Span & $0.37 \mathrm{~m}$ \\
Surface Area & $0.085 \mathrm{~m}^{2}$ \\
Aspect ratio & 3.34 \\
Thickness Ratio & $11 \%-36 \%$ \\
\hline
\end{tabular}

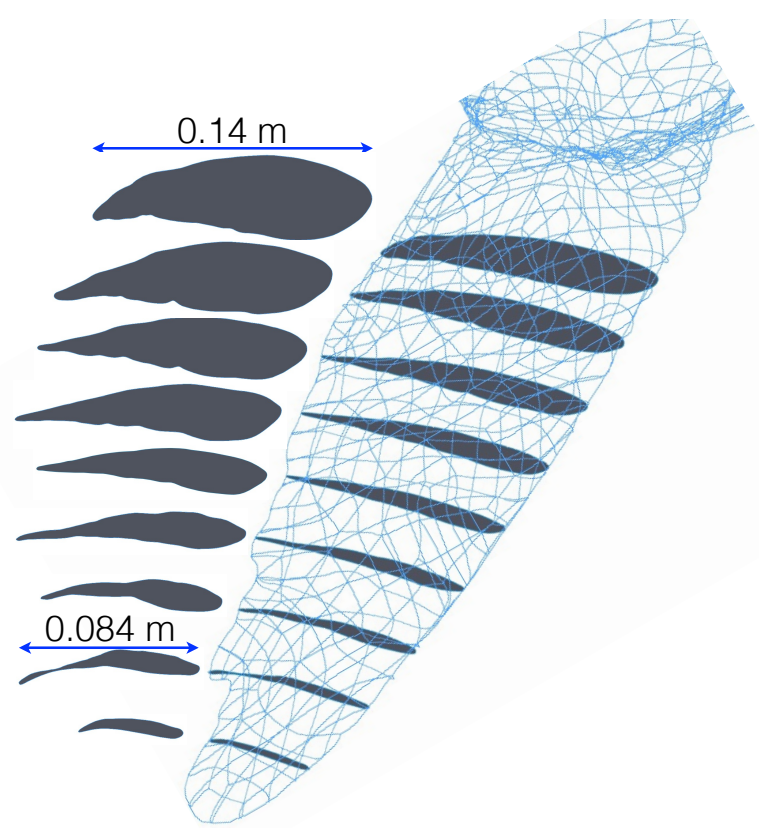

Figure 4: Flipper mesh view with extracted.

\section{CONCLUSIONS}

Three noninvasive scanning techniques were used to extract the geometry of the California sea lion foreflipper. This paper highlights relative advantages and disadvantages of each method (resolution, cost, required proficiency, etc.). The scanned flipper results are qualitatively compared for geometric flipper features such as trailing edge shape, vein marks, and overall noise in the 3D model. Of the three methods, the high resolution structured light scanner was found to be significantly more accurate than either the Kinect based scanner or the image based method. The results using the high resolution scanner present a high quality model of the flipper, and eliminates the need for post processing scaling. However, the level of proficiency and the price associated with this method is significantly higher. For the purposes of hydrodynamic research, the required level of details justifies the use of the high resolution scanner and prohibits the use of the lower accuracy models obtained using the other two methods. The two lower details methods did not present with sufficient details and surface smoothness to allow even a general approximate hydrodynamic design that would be based on the sea lion flipper geometry.

\section{REFERENCES}

[1] U. K. Müller, J. Smit, E. J. Stamhuis, , and J. J. Videler. How the body contributes to the wake in undulatory fish swimming flow fields of a swimming eel (anguilla anguilla). The Journal of Experimental Biology, 204(16):2751-2762, 2001.

[2] M. Sfakiotakis, D. M. Lane, and J. B. C. Davies. Review of fish swimming modes for aquatic locomotion. IEEE Journal of Oceanic Engineering, 24(2):237-252, 1999. 
[3] Steven D. Feldkamp. Swimming in the california sea lion: Morphometrics, drag and energetics. Journal of Experimental Biology, 131:117-135, 1987.

[4] L. L. Stelle, R.W. Blake, and Trites A. W. Hydrodynamic drag in steller sea lions (eumetopias jubatus). The Journal of Experimental Biology, 203:1915-1923, 2000.

[5] Steven J. Godfrey. Additional observations of subaqueous locomotion in the california sea lion (zalophus californianus). Aquatic Mammals, 11(2):53-57, 1985.

[6] F. E. Fish. Transitions from drag-based to lift-based propulsion in mammalian swimming. American Zoologist, 36(6):628-641, 1996.

[7] Frank E. Fish, J. Hurley, and Daniel P. Costa. Maneuverability by the sea lion zalophus californianus: turning performance of an unstable body design. The Journal of Experimental Biology, 206:667-674, 2003.

[8] S. D. Feldkamp. The effects of net entanglement on the drag and power output of a california sea lion, zalophus californianus. Fishery Bulletin, 83(4):692-694, 1985.
[9] C. Friedman and M. Leftwich. The kinematics of the california sea lion foreflipper during forward swimming. Bioinspiration and Biomimetics, 9(4):046010, 2014.

[10] R. A. Newcombe, A. J. Davison, S. Izadi, P. Kohli, O. Hilliges, J. Shotton, D. Molyneaux, S. Hodges, D. Kim, and A. Fitzgibbon. Kinectfusion: Real-time dense surface mapping and tracking. In Mixed and augmented reality (ISMAR), $10^{\text {th }}$ IEEE international symposium on, pages 127-136, 2011.

[11] D. Scharstein and R. Szeliski. High-accuracy stereo depth maps using structured light. In In Computer Vision and Pattern Recognition, Proceedings of the IEEE Computer Society Conference on, volume 1, pages 1195, June 2003.

[12] W. Fassman. An experimental study of bio-inspired force generation by unsteady flow features. Master's thesis, ME Department, Brigham Young University, May 2014. 